$14^{\text {th }}$ INTERNATIONAL WORKSHOP ON RADIATION IMAGING DETECTORS, 1-5 JULY 2012, FIgUEIRA DA FOZ, PORTUGAL

\title{
Energy calibration of a CdTe X-ray pixel sensor hybridized to a Timepix chip
}

\section{Ponchut ${ }^{1}$ and M. Ruat}

European Synchrotron Radiation Facility,

6 rue Jules Horowitz, F-38000 Grenoble, France

E-mail: ponchut@esrf.fr

ABSTRACT: The X-ray energy response of a CdTe pixel sensor was investigated in view of applications for synchrotron experiments at high energy. We performed an energy calibration of a $1 \mathrm{~mm}$ thick CdTe pixel sensor of $55 \mu \mathrm{m}$ pixel pitch bump-bonded to a Timepix chip in the 6-120 keV range. Calibration data were acquired in time-over-threshold (TOT) mode with monochromatic synchrotron beams of different energies and with gamma ray sources. Charge sharing in the raw TOT X-ray images was on-line corrected with a cluster summing algorithm implemented in the acquisition software. Variation of the TOT signal with respect to incident X-ray energy was found to be linear from $8 \mathrm{keV}$ to $120 \mathrm{keV}$. A raw energy resolution of $43 \% \mathrm{FWHM}$ was achieved at $59.5 \mathrm{keV}$ without prior per-pixel TOT calibration. Fluorescence of the CdTe material was identified as the main drawback of fine pitch CdTe pixel sensors if considering spectral X-ray imaging applications at high energy.

KEYWORDS: X-ray detectors; Hybrid detectors

\footnotetext{
${ }^{1}$ Corresponding author.
} 


\section{Contents}

1 Introduction 1

2 Energy calibration methodology in TOT mode 1

3 Experimental method and results $\quad 2$

3.1 Energy spectrum measurement 2

3.2 Energy calibration 3

4 Conclusion 3

\section{Introduction}

Photon-counting pixel detectors based on CdTe or other high-Z sensor materials cumulate the advantages of an X-ray energy range extended beyond $100 \mathrm{keV}$, a high spatial resolution, and a noiseless detection. Evaluations of CdTe pixel sensors coupled to Timepix [1] readout chip have confimed the great interest of such devices in particular for synchrotron beamlines specialized in material science or X-ray imaging [2]. In addition to photon counting mode, the Timepix chip can be operated in Time-Over-Threshold (TOT) mode giving access to energy-sensitive imaging [3]. Our present work consisted in calibrating the energy response of a CdTe/Timepix device in TOT mode over a wide X-ray energy range and in evaluating its spectroscopic capabilities, in view of potential applications to energy-resolved imaging on high-energy synchrotron beamlines.

\section{Energy calibration methodology in TOT mode}

In TOT mode the pixel counter is incremented at each clock cycle as long as the input charge signal remains above threshold (figure 1). Since the charge signal below threshold does not contribute to the TOT signal the pixel response with respect to input charge becomes non-linear at low energy [1, 3]. A calibration is therefore necessary in order to retrieve the input charge value and thus the incident X-ray energy. Due to charge sharing, calibration of a given pixel requires to retain only spatially isolated single-pixel X-ray events occuring in that pixel [3]. However such method is difficult to apply in our case because due to a more important charge sharing with a fine pitch 1 $\mathrm{mm}$ thick CdTe sensor, occurence of single pixel events is lower than with thin silicon sensors as used in [3].

In the frame of our study, we will therefore assume that measurements of TOT signals per Xray event statistically averaged over a region of interest of the detector matrix enables us to reach sufficient precision for energy calibration even if not correcting the dispersion of the individual pixels TOT responses. 


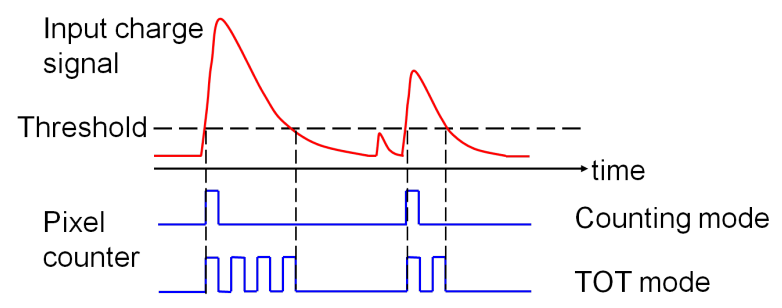

Figure 1. Timepix TOT mode principle.
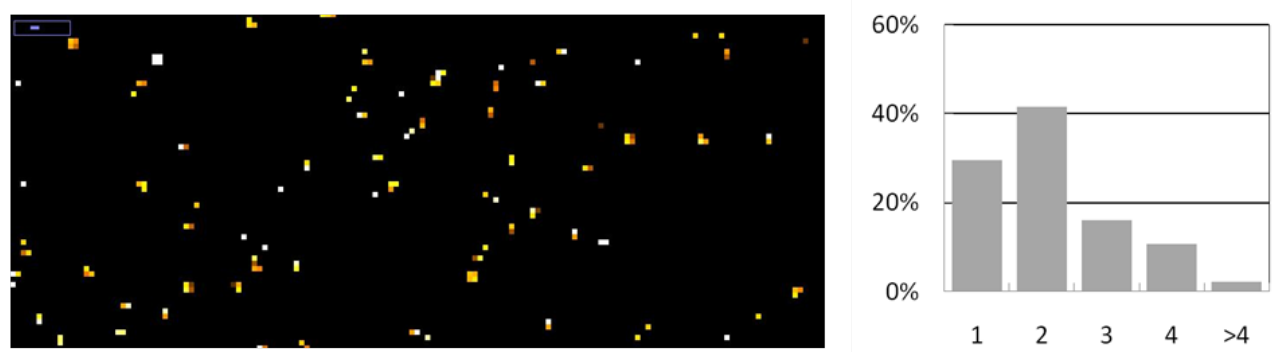

Figure 2. Left: detail of a raw image of isolated X-ray events forming clusters of one to four pixels Right: typical cluster size distribution.

\section{Experimental method and results}

We use a CdTe/Timepix assembly manufactured by XIE (Freiburg) and consisting of a monolithic CdTe pixel sensor bump-bonded to four Timepix chips in $2 \times 2$ arrangement. The detection area is $28 \times 28 \mathrm{~mm}^{2}$ with $512 \times 512$ pixels at $55 \times 55 \mu \mathrm{m}$ pitch. The CdTe sensor is biased at $-400 \mathrm{~V}$. The assembly is mounted in a MAXIPIX [4] detector system. MAXIPIX achieves simultaneous readout of the four Timepix chips with $290 \mu$ s readout dead time and up to 350 frames/s acquisition rate.

In order to enable the processing of individual X-ray events the frame exposure time must be set so as to avoid an overlap of individual pixel clusters (figure 2). Given the MAXIPIX dead time characteristics, this limits the maximum local count rate to typically 30 incident X-rays/pixel/s for $1 \mathrm{~ms}$ exposures.

The raw TOT frames are first flat-field corrected using a high statistics flatfield image acquired in TOT mode. This performs a first-order correction of the dispersion of individual pixels TOT response at the incident X-ray energy. Then a charge sharing correction is performed by generating an image of the integral values of each cluster reallocated to the pixel nearest to the cluster centroïd. Both operations are implemented as an on-line processing in the acquisition software.

\subsection{Energy spectrum measurement}

The detector is exposed to a ${ }^{241} \mathrm{Am}$ gamma source filtered with a $\mathrm{Cu}$ foil. 250 images of 100 $\mathrm{ms}$ exposure are acquired with online flatfield and cluster processing. The main ${ }^{241} \mathrm{Am}$ peak $(59.5$ $\mathrm{keV}$ ) and the $\mathrm{Cu}$ fluorescence peak $(8 \mathrm{keV})$ can be identified (figure 3$)$. The intense peak in between merges the signals from CdTe fluorescence lines and from the corresponding escapes, all contained in the $23.2-36.3 \mathrm{keV}$ range at the $59.5 \mathrm{keV}$ excitation energy. 


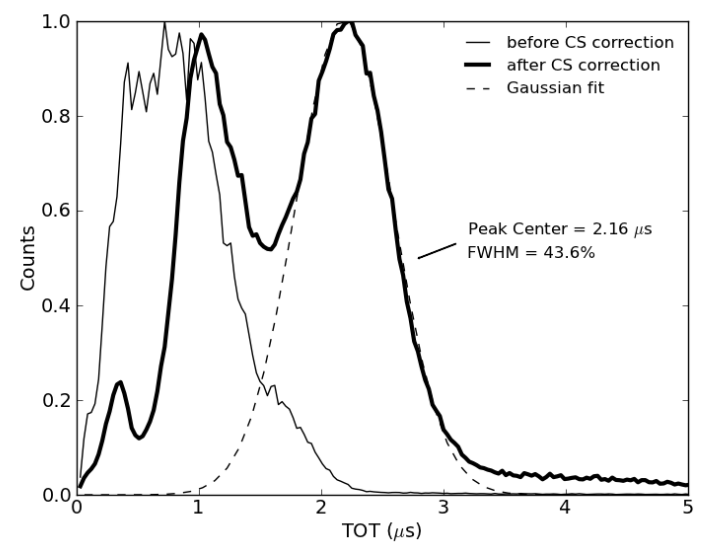

Figure 3. Histogram of raw (thin line) and processed (bold line) TOT frames. The TOT units represent the actual time over threshold obtained as the product of the pixel value by the chip clock period (20 ns).

The FWHM of the main peak at $59.5 \mathrm{keV}$ is about $43 \%$. A slightly better energy resolution is reported on a similar CdTe/Timepix device after per-pixel TOT calibration [5]. An even better energy resolution is achieved with a silicon pixel sensor [3], but this is at the expense of losing a factor 100 in detection efficiency at this energy if compared to our CdTe sensor.

The important fluorescence effect must be regarded as an intrinsic property of the $55 \mu \mathrm{m}$ pitch CdTe pixel sensor. This prevents an efficient reconstruction of incident X-ray spectrum and can therefore limit the applications of fine pitch CdTe sensors for energy-resolved X-ray imaging at high energy. To be noted, results obtained in $[5,6]$ suggest that this effect is reduced with a pixel size of $110 \mu \mathrm{m}$ instead of $55 \mu \mathrm{m}$.

\subsection{Energy calibration}

Acquisitions in cluster-processed TOT mode are performed at different energies: monochromatic synchrotron beam at SOLEIL $(6,10,15,20$, and $25 \mathrm{keV})$, irradiation with ${ }^{55} \mathrm{Fe}(5.9 \mathrm{keV}),{ }^{109} \mathrm{Cd}$ $(22-25 \mathrm{keV}),{ }^{241} \mathrm{Am}(59.5 \mathrm{keV})$ and ${ }^{57} \mathrm{Co}(122 \mathrm{keV})$ gamma ray sources at ESRF and CEA-LETI. Photopeaks in the image histograms are identified and fitted with Gaussians, and the TOT values corresponding to the peaks center positions are extracted as a function of the incident photon energy (figure 4). These data can be accurately fitted using the same surrogate function as that used in [3] for individual pixels calibration. The calibration function is linear up to at least $120 \mathrm{keV}$, except in the energy range close to the threshold (here set at $4.95 \mathrm{keV}$ ).

\section{Conclusion}

The energy response of a $55 \mu \mathrm{m}$ pixel pitch CdTe sensor measured with Timepix in TOT mode was found to be linear from about $8 \mathrm{keV}$ to at least $120 \mathrm{keV}$. Energy-encoded X-ray images were obtained after online charge sharing correction of the raw TOT images. This demonstrated the possibility of spectral imaging at $55 \mu \mathrm{m}$ spatial resolution in a wide energy range using CdTe pixel sensors, with however a detrimental effect of the CdTe material X-ray fluorescence. 


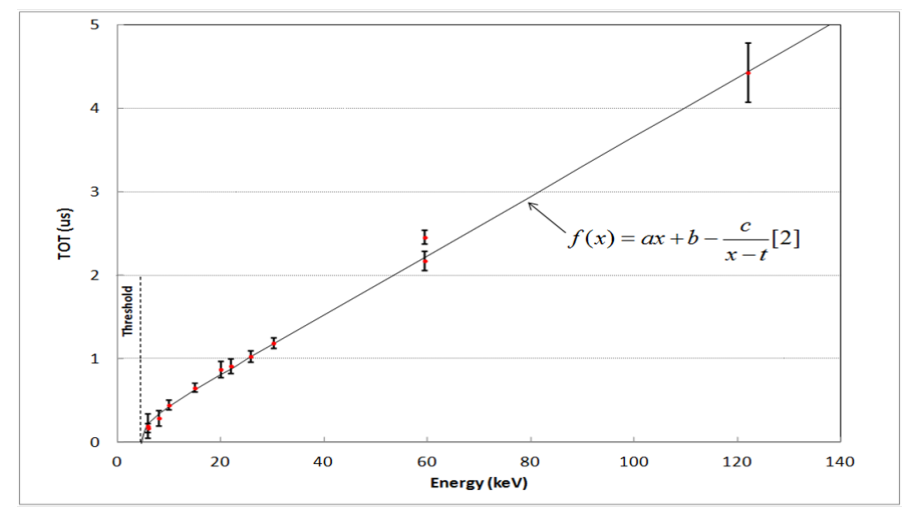

Figure 4. Energy calibration in TOT mode with cluster-summing applied.

\section{Acknowledgments}

This work received funding from ELISA, a European project of the 7th Framework program, grant agreement $n^{\circ}$ 226716. The Timepix chip is developed by CERN in the frame of Medipix 2 collaboration. We thank Stéphanie Hustache, Sylvain Ravy and Kadda Medjoubi for beamtime and support at SOLEIL "Cristal" beamline, Eric Gros d'Aillon and Guillaume Montemont at CEALETI for tests with X-ray sources.

\section{References}

[1] X. Llopart et al.,Timepix, a 65k programmable pixel readout chip for arrival time, energy and/or photon counting measurements, Nucl. Instrum. Meth. A 581 (2007) 485.

[2] M. Ruat et al., Characterization of a pixelated CdTe X-ray detector using the Timepix photon-counting readout chip, IEEE Trans. Nucl. Sci. 59 (2012) 2392.

[3] J. Jakubek, Pixel detectors for imaging with heavy charged particles, Nucl. Instrum. Meth. A 591 (2008) 155.

[4] C. Ponchut et al., MAXIPIX, a fast readout photon-counting X-ray area detector for synchrotron applications, 2011 JINST 6 C01069.

[5] D. Maneuski et al., Imaging and spectroscopic performance studies of pixellated CdTe Timepix detector, 2011 JINST 7 C01038.

[6] E. Fröjdh et al., Depth of interaction and bias voltage dependence of the spectral response in a pixellated CdTe detector operating in time-over-threshold mode subjected to monochromatic X-rays, 2011 JINST 7 C03002. 